QUARTERLY OF APPLIED MATHEMATICS

VOLUME LXX, NUMBER 1

MARCH 2012, PAGES 111-132

S 0033-569X(2011)01243-0

Article electronically published on August 26, 2011

\title{
WELL-POSEDNESS AND REGULARITY FOR NON-UNIFORM SCHRÖDINGER AND EULER-BERNOULLI EQUATIONS WITH BOUNDARY CONTROL AND OBSERVATION
}

BY

BAO-ZHU GUO (School of Mathematical Sciences, Shanxi University, Taiyuan 030006, People's Republic of China, Academy of Mathematics and Systems Science, Academia Sinica, Beijing 100190, People's Republic of China, and School of Computational and Applied Mathematics, University of the Witwatersrand, Wits 2050, Johannesburg, South Africa)

AND

ZHI-CHAO SHAO (School of Information Technology and Management and China Service Industry Research Center, University of International Business and Economics, Beijing 100029, People's Republic of China)

Abstract. The open-loop systems of a Schrödinger equation and an Euler-Bernoulli equation with variable coefficients and boundary controls and collocated observations are considered. It is shown, with the help of a multiplier method on a Riemannian manifold, that both systems are well-posed in the sense of D. Salamon and regular in the sense of G. Weiss. The feed-through operators are found to be zero. The results imply particularly that the exact controlability of each open-loop system is equivalent to the exponential stability of the associated closed-loop system under the output proportional feedback.

1. Introduction and main results. Well-posed and regular linear infinite dimensional systems first introduced by D. Salamon and G. Weiss in the 1980s ([25, 26, 29, 30, ) are a quite general class of linear infinite-dimensional systems. They cover many control systems described by time delay equations and partial differential equations with actuators and sensors supported at isolated points, subdomains, or on a part of the boundary

Received August 9, 2010.

2000 Mathematics Subject Classification. Primary 35L35, 93C20, 93D15.

Key words and phrases. Schrödinger equation, Euler-Bernoulli equation, well-posed and regular system, variable coefficients, boundary control and observation.

This work was carried out with the support of the National Natural Science Foundation of China, the National Research Foundation of South Africa, and the National Basic Research Program of China (973 Program: 2011CB808002).

Zhi-Chao Shao also acknowledges the support of the Scientific Research Foundation for the Returned Overseas Scholar by Education Ministry of China and the program for Innovative Research Team in UIBE.

E-mail address: bzguo@iss.ac.cn

E-mail address: zcshao@amss.ac.cn

(C)2011 Brown University 
of the spatial region [6]. This class of linear infinite-dimensional systems, although the input and output operators are usually unbounded, may possess many properties paralleling in many ways to finite-dimensional systems, for instance, representation, transfer function, internal model based tracking and disturbance rejection, stabilizing controller parametrization, and quadratic optimal control, to name just a few [7.

The abstract theory of well-posed and regular linear systems has been quite fruitful. However, only a few multidimensional PDEs have been verified to be well-posed and regular. For the systems with constant coefficients, the well-posedness and regularity of a multidimensional heat equation with both Dirichlet and Neumann type boundary controls were established in [5], and that for a wave equation with boundary Dirichlet input and collocated output was proved in [1] and [14]. The well-posedness and regularity for multi-dimensional Schrödinger and Euler-Bernoulli equations were reported in [11, 12, 19. The well-posedness and regularity for a wave equation with variable coefficients and Dirichlet boundary control and observation was established in [15], and that for a plate equation with variable coefficients under Neumann boundary control and observation was obtained in [16]. In [13, the exact controllability, well-posedness and regularity for the transmission problem of plate equations with variable coefficients and Neumann boundary control and observation were considered. Recently, the well-posedness and regularity of linear elasticity with Dirichlet boundary control and collocated observation were established in [3, 17.

The objective of this paper is, on the one hand, to generalize the well-posedness for a Schrödinger equation with Dirichlet boundary control [19] and an Euler-Bernoulli plate equation with boundary moment control [22], all with collocated observation, to the variable coefficients cases, and on the other hand, to show that, in both cases, the systems are regular with zero feed-through operators.

The systems to be considered are described, respectively, by the Schrödinger equation with Dirichlet boundary control and collocated observation

$$
\left\{\begin{array}{l}
v_{t}(x, t)-i P v(x, t)=0, x=\left(x_{1}, x_{2}, \ldots, x_{n}\right) \in \Omega, t>0, \\
v(x, t)=u(x, t), x \in \Gamma, t \geq 0 \\
y(x, t)=-i \frac{\partial \mathcal{A}^{-1} v(x, t)}{\partial \nu_{\mathcal{A}}}, x \in \Gamma, t \geq 0
\end{array}\right.
$$

and the Euler-Bernoulli equation with boundary moment control and collocated observation

$$
\left\{\begin{array}{l}
w_{t t}(x, t)+P^{2} w(x, t)=0, x \in \Omega, t>0, \\
w(x, t)=0, x \in \Gamma, t \geq 0 \\
P w(x, t)=u(x, t), x \in \Gamma, t \geq 0 \\
y(x, t)=\frac{\partial \mathcal{A}^{-1} w_{t}(x, t)}{\partial \nu_{\mathcal{A}}}, x \in \Gamma, t \geq 0
\end{array}\right.
$$


where $\Omega \subset \mathbb{R}^{n}(n \geq 2)$ is an open bounded region with $C^{2}$-boundary $\Gamma$. $P$ is a second order partial differential operator

$$
P=-\sum_{i, j=1}^{n} \frac{\partial}{\partial x_{i}}\left(a_{i j}(x) \frac{\partial}{\partial x_{j}}\right)
$$

and for some constants $a, b>0$,

$$
\begin{gathered}
a \sum_{i=1}^{n}\left|\xi_{i}\right|^{2} \leq \sum_{i, j=1}^{n} a_{i j}(x) \xi_{i} \overline{\xi_{j}} \leq b \sum_{i=1}^{n}\left|\xi_{i}\right|^{2}, \forall x \in \bar{\Omega}, \xi=\left(\xi_{1}, \xi_{2}, \ldots, \xi_{n}\right) \in \mathbb{C}^{n}, \\
a_{i j}=a_{j i} \in C^{\infty}\left(\mathbb{R}^{n}\right), \forall i, j=1,2, \ldots, n .
\end{gathered}
$$

The operator $\mathcal{A}$ is defined by

$$
\mathcal{A} f:=P f, \forall f \in D(\mathcal{A})=H^{2}(\Omega) \cap H_{0}^{1}(\Omega)
$$

and

$$
\frac{\partial}{\partial \nu_{\mathcal{A}}} \equiv \nu_{\mathcal{A}}=\sum_{i, j=1}^{n} a_{i j} \nu_{j} \frac{\partial}{\partial x_{i}},
$$

where $\nu=\left(\nu_{1}, \nu_{2}, \ldots, \nu_{n}\right)$ is the unit normal of $\partial \Omega$ pointing towards the exterior of $\Omega$. $u$ and $y$ are the input functions (or controls) and the output functions (or observations), respectively. It is seen that both operators $\mathcal{A}$ and $P$ appear in the system (1.1) and (1.2) simultaneously. That is why we put them together and treat the two with a similar approach.

Let $H=H^{-1}(\Omega), \mathcal{H}=H_{0}^{1}(\Omega) \times H^{-1}(\Omega)$ and $U=L^{2}(\Gamma)$. Theorem 1.1 is the generalization of Proposition 4.2 of [19] where the coefficients of the system (1.1) are constants.

Theorem 1.1. Let $T>0, v_{0} \in H$ and $u \in L^{2}(0, T ; U)$. Then there exists a unique solution $v \in C(0, T ; H)$ to the Equation (1.1) satisfying the initial condition $v(\cdot, 0)=v_{0}$. Moreover, there exists a constant $C_{T}>0$, independent of $\left(v_{0}, u\right)$, such that

$$
\|v(\cdot, T)\|_{H}^{2}+\|y\|_{L^{2}(0, T ; U)}^{2} \leq C_{T}\left[\left\|v_{0}\right\|_{H}^{2}+\|u\|_{L^{2}(0, T ; U)}^{2}\right] .
$$

Theorem 1.2 below generalizes the well-posedness result of Theorem 4.29 of [22] to the variable coefficients case.

Theorem 1.2. Let $T>0,\left(w_{0}, w_{1}\right) \in \mathcal{H}$, and $u \in L^{2}(0, T ; U)$. Then there exists a unique solution $\left(w, w_{t}\right) \in C([0, T] ; \mathcal{H})$ to the Equation (1.2) satisfying $w(\cdot, 0)=w_{0}$ and $w_{t}(\cdot, 0)=w_{1}$. Moreover, there exists a constant $C_{T}>0$, independent of $\left(w_{0}, w_{1}, u\right)$, such that

$$
\left\|\left(w(\cdot, T), w_{t}(\cdot, T)\right)\right\|_{\mathcal{H}}^{2}+\|y\|_{L^{2}(0, T ; U)}^{2} \leq C_{T}\left[\left\|\left(w_{0}, w_{1}\right)\right\|_{\mathcal{H}}^{2}+\|u\|_{L^{2}(0, T ; U)}^{2}\right] .
$$

Theorems 1.1 and 1.2 imply that the open-loop systems (1.1) and (1.2) are well-posed in the sense of D. Salamon with the state spaces $H$ and $\mathcal{H}$, respectively, with input and output space $U$ [27]. These results together with Theorem 2.2 of [2] and Proposition 3.1 of 22] (see also Theorem 3 of [10]) give immediately the following corollary. 
Corollary 1.3. The systems (1.1) or (1.2) are exactly controllable in some time interval $[0, T]$ if and only if the corresponding closed-loop system under the proportional output feedback $u=-k y, k>0$ are exponentially stable.

For the exponential stability of the system (1.1) under the feedback $u=-k y, k>0$, we refer to [24]. For the exact controllability of the system (1.2), we refer the reader to [34] and 20.

Theorem 1.4. The system (1.1) is regular. More precisely, if $v(\cdot, 0)=0$ and $u(\cdot, t) \equiv$ $u(\cdot) \in U$ is a step input, then the corresponding step response $y$ satisfies

$$
\lim _{\sigma \rightarrow 0+} \int_{\Gamma}\left|\frac{1}{\sigma} \int_{0}^{\sigma} y(x, t) d t\right|^{2} d x=0 .
$$

Theorem 1.5. The system (1.2) is regular. More precisely, if $w(\cdot, 0)=w_{t}(\cdot, 0)=0$ and $u(\cdot, t) \equiv u(\cdot) \in U$ is a step input, then the corresponding output $y$ satisfies

$$
\lim _{\sigma \rightarrow 0+} \int_{\Gamma}\left|\frac{1}{\sigma} \int_{0}^{\sigma} y(x, t) d t\right|^{2} d x=0 .
$$

Theorems 1.1, 1.2, 1.4 and 1.5 together show that the systems (1.1) and (1.2) are wellposed regular linear systems with feedthrough operators zero. This makes the systems (1.1) and (1.2) parallel in many ways to linear finite-dimensional ones.

Our main approach in proving above mentioned results is the Riemannian geometry method introduced in 33 for the proof of exact controllability of wave equation with variable coefficients. The difficulty of our proofs also arises from the occurrence of the variable coefficients as that in [33. By the geometry approach, similar to [34, the computations on the Riemannian manifold become much more simple, which it would be extremely tedious and even hardly carried out in Euclidean space. The key point is that by the introduction of the Riemannian metric, the corresponding differential operators with variable coefficients in Euclidean space become the differential operators with constant coefficients on the Riemannian manifold, except some low order perturbations. Therefore, the techniques in dealing with problems with constant coefficients in Euclidean space can then be moved to Riemannian manifold. From this point view, it seems that the Riemannian geometry method becomes natural in dealing with the non-uniform problems.

The remainder of the paper is organized as follows. In Section 2, the systems (1.1) and (1.2) are casted into the abstract settings separately, and some basic background on Riemannian geometry is introduced. The proofs of Theorems 1.1 and 1.2 are given in Section 3 and Section 4, respectively. Section 5 is devoted to the proofs of Theorems 1.4 and 1.5 .

2. Abstract formulations and preliminaries. In order to formulate the system (1.1) and (1.2) into abstract forms, we need some notation and facts in Riemannian geometry. 
By the ellipticity condition (1.3), we let $G(x)$ and $\rho(x)$ be the following positive matrix and its determinant, respectively,

$$
G(x):=\left[g_{i j}(x)\right]_{n \times n}=\left[a_{i j}(x)\right]_{n \times n}^{-1}, \quad \rho(x):=\operatorname{det}\left[g_{i j}(x)\right]_{n \times n}, \forall x \in \mathbb{R}^{n} .
$$

For each $x=\left(x_{1}, x_{2}, \ldots, x_{n}\right) \in \mathbb{R}^{n}$, define the inner product and norm over the tangent space $\mathbb{R}_{x}^{n}$ of $\mathbb{R}^{n}$ by

$$
\begin{aligned}
& \langle X, Y\rangle_{g}:=\sum_{i, j=1}^{n} g_{i j} \alpha_{i} \beta_{j}, \\
& |X|_{g}:=\langle X, X\rangle_{g}^{1 / 2}, \forall X=\sum_{i=1}^{n} \alpha_{i} \frac{\partial}{\partial x_{i}}, Y=\sum_{i=1}^{n} \beta_{i} \frac{\partial}{\partial x_{i}} \in \mathbb{R}_{x}^{n} .
\end{aligned}
$$

Then $\left(\mathbb{R}^{n}, g\right)$ becomes a Riemannian manifold with Riemannian metric $g$ [34. Denote by $D$ the Levi-Civita connection with respect to $g$. Let $N$ be a smooth vector field on $\left(\mathbb{R}^{n}, g\right)$. Then for each $x \in \mathbb{R}^{n}$, the covariant differential $D N$ of $N$ determines a bilinear form on $\mathbb{R}_{x}^{n}$,

$$
D N(X, Y)=\left\langle D_{X} N, Y\right\rangle_{g}, \forall X, Y \in \mathbb{R}_{x}^{n},
$$

where $D_{X} N$ stands for the covariant derivative of the vector field $N$ with respect to $X$.

For any $\varphi \in C^{2}\left(\mathbb{R}^{n}\right)$ and $N=\sum_{i=1}^{n} h^{i}(x) \frac{\partial}{\partial x_{i}}$, denote

$$
\begin{aligned}
& \operatorname{div}_{0}(N):=\sum_{i=1}^{n} \frac{\partial h^{i}}{\partial x_{i}}, D \varphi:=\nabla_{g} \varphi=\sum_{i, j=1}^{n} a_{i j} \frac{\partial \varphi}{\partial x_{j}} \frac{\partial}{\partial x_{i}}, \\
& \operatorname{div}_{g}(N):=\sum_{i=1}^{n} \rho^{-1 / 2} \frac{\partial}{\partial x_{i}}\left(\rho^{1 / 2} h^{i}\right), \\
& \Delta_{g} \varphi:=\sum_{i, j=1}^{n} \rho^{-1 / 2} \frac{\partial}{\partial x_{i}}\left(\rho^{1 / 2} a_{i j} \frac{\partial \varphi}{\partial x_{j}}\right)=-P \varphi+(D q) \varphi, q(x)=1 / 2 \log (\rho(x)),
\end{aligned}
$$

where $\operatorname{div}_{0}$ is the divergence operator in Euclidean space $\mathbb{R}^{n}$, and $\nabla_{g}, \operatorname{div}_{g}$ and $\Delta_{g}$ are the gradient operator, the divergence operator and the Beltrami-Laplace operator in $\left(\mathbb{R}^{n}, g\right)$, respectively.

Denote by $\mu=\frac{\nu_{\mathcal{A}}}{\left|\nu_{\mathcal{A}}\right|_{g}}$ the unit outward-pointing normal to $\partial \Omega$ in terms of the Riemannian metric $g$. The following Lemma 2.1, providing some useful identities 28, pp. $128,138]$, will be used frequently in subsequent sections.

Lemma 2.1. Let $\varphi, \psi \in C^{1}(\bar{\Omega})$ and $N$ be a vector field on $\left(\mathbb{R}^{n}, g\right)$. Then we have the following.

(1) Divergence formulae and theorems:

$$
\begin{gathered}
\operatorname{div}_{0}(\varphi N)=\varphi \operatorname{div}_{0}(N)+N(\varphi), \quad \operatorname{div}_{g}(\varphi N)=\varphi \operatorname{div}_{g}(N)+N(\varphi), \\
\int_{\Omega} \operatorname{div}_{0}(N) d x=\int_{\Gamma} N \cdot \nu d \Gamma, \quad \int_{\Omega} \operatorname{div}_{g}(N) d x=\int_{\Gamma}\langle N, \mu\rangle_{g} d \Gamma .
\end{gathered}
$$


(2) Green's first formulae:

$$
\begin{aligned}
& \int_{\Omega} \psi P \varphi d x=-\int_{\Gamma} \psi \frac{\partial \varphi}{\partial \nu_{\mathcal{A}}} d \Gamma+\int_{\Omega}\left\langle\nabla_{g} \varphi, \nabla_{g} \psi\right\rangle_{g} d x \\
& \int_{\Omega} \psi \Delta_{g} \varphi d \Omega=\int_{\Gamma} \psi \frac{\partial \varphi}{\partial \mu} d S-\int_{\Omega}\left\langle\nabla_{g} \varphi, \nabla_{g} \psi\right\rangle_{g} d \Omega
\end{aligned}
$$

(3) Green's second formulae:

$$
\begin{gathered}
\int_{\Omega} \psi P \varphi d x-\int_{\Omega} P \psi \varphi d x=-\int_{\Gamma} \psi \frac{\partial \varphi}{\partial \nu_{\mathcal{A}}} d \Gamma+\int_{\Gamma} \frac{\partial \psi}{\partial \nu_{\mathcal{A}}} \varphi d \Gamma, \\
\int_{\Omega} \psi \Delta_{g} \varphi d \Omega-\int_{\Omega} \Delta_{g} \psi \varphi d \Omega=\int_{\Gamma} \psi \frac{\partial \varphi}{\partial \mu} d S-\int_{\Gamma} \frac{\partial \psi}{\partial \mu} \varphi d \Gamma,
\end{gathered}
$$

where $d \Omega$ and $d S$ are the canonical volume elements on $\bar{\Omega}$ and $\Gamma$, respectively.

Similar to (4) of Lemma 2.1 in [33], we have the following multiplier identity.

Lemma 2.2. Let $N$ be a smooth real vector field on $\left(\mathbb{R}^{n}, g\right)$. For $z \in C^{1}(\bar{\Omega})$, the following formula holds:

$$
\left\langle\nabla_{g} z, \nabla_{g}(N(z))\right\rangle_{g}=D N\left(\nabla_{g} z, \nabla_{g} z\right)+\frac{1}{2} \operatorname{div}_{g}\left(\left|\nabla_{g} z\right|_{g}^{2} N\right)-\frac{1}{2}\left|\nabla_{g} z\right|_{g}^{2} \operatorname{div}_{g}(N) .
$$

The following Lemma 2.3 is straightforward.

LEMma 2.3. Let $\varphi$ be a complex function defined on $\bar{\Omega}$ with suitable regularity. Then there exist some constants $C$ 's possibly depending on $g, N$ and $\Omega$, such that

(1)

$$
\begin{aligned}
& \sup _{x \in \bar{\Omega}}|N|_{g} \leq C, \quad \sup _{x \in \bar{\Omega}}|D N|_{g} \leq C, \quad \sup _{x \in \bar{\Omega}}\left|\operatorname{div}_{g}(N)\right| \leq C, \quad \sup _{x \in \bar{\Omega}}|D q|_{g} \leq C, \\
& \sup _{x \in \bar{\Omega}}\left|\nabla_{g}\left(\operatorname{div}_{g} N\right)\right|_{g} \leq C
\end{aligned}
$$

$$
\begin{aligned}
& |N(\varphi)| \leq C\left|\nabla_{g} \varphi\right|_{g}, \quad|D q(\varphi)| \leq C\left|\nabla_{g} \varphi\right|_{g}, \quad\left|D N\left(\nabla_{g} \varphi, \nabla_{g} \bar{\varphi}\right)\right| \leq C\left|\nabla_{g} \varphi\right|_{g}^{2} \\
& \left|\left\langle\nabla_{g} \varphi, \nabla_{g}\left(\operatorname{div}_{g} N\right)\right\rangle_{g}\right| \leq C\left|\nabla_{g} \varphi\right|_{g}
\end{aligned}
$$

where $q(x)=1 / 2 \log (\rho(x))$ and $\rho$ is given by (2.1);

$$
\int_{\Omega}|\varphi|^{2} d x \leq C\|\varphi\|_{H^{1}(\Omega)}^{2}, \int_{\Omega}\left|\nabla_{g} \varphi\right|_{g}^{2} d x \leq C\|\varphi\|_{H^{1}(\Omega)}^{2}, \int_{\Omega}\left|\nabla_{g}(P \varphi)\right|_{g}^{2} d x \leq C\|\varphi\|_{H^{3}(\Omega)}^{2} .
$$

Now we are in a position to cast the system (1.1) into an abstract form of a first-order system in the Hilbert state space $H=H^{-1}(\Omega)$.

By Green's first formula, an extension $\mathscr{A}_{1} \in \mathcal{L}\left(H_{0}^{1}(\Omega), H^{-1}(\Omega)\right)$ of $\mathcal{A}$ can be defined by

$$
\left\langle\mathscr{A}_{1} \varphi, \psi\right\rangle_{H^{-1}(\Omega) \times H_{0}^{1}(\Omega)}=\int_{\Omega}\left\langle\nabla_{g} \varphi, \nabla_{g} \phi\right\rangle_{g} d x, \forall \varphi, \psi \in H_{0}^{1}(\Omega) .
$$


Then $\mathscr{A}_{1}$ is a positive self-adjoint operator in $H$, and by means of the Lax-Milgram theorem, $\mathscr{A}_{1}$ is a canonical isomorphism from $D\left(\mathscr{A}_{1}\right)=H_{0}^{1}(\Omega)$ onto $H$. It is easily shown (see e.g., [18) that $D\left(\mathscr{A}_{1}^{1 / 2}\right)=L^{2}(\Omega)$ and $\mathscr{A}_{1}^{1 / 2}$ is a canonical isomorphism from $L^{2}(\Omega)$ onto $H$.

Define the Dirichlet map $\Upsilon \in \mathcal{L}\left(L^{2}(\Gamma), H^{1 / 2}(\Omega)\right.$ ) (21] p. 189) as follows:

$$
\Upsilon u=\varphi \Longleftrightarrow\left\{\begin{array}{l}
P \varphi=0 \text { in } \Omega, \\
\left.\varphi\right|_{\Gamma}=u .
\end{array}\right.
$$

Then (1.1) can be written as

$$
\dot{v}-i \mathscr{A}_{1}(v-\Upsilon u)=0 .
$$

Identifying $H$ with its dual $H^{\prime}$, we have the following relations:

$$
D\left(\mathscr{A}_{1}\right) \subset D\left(\mathscr{A}_{1}^{1 / 2}\right) \hookrightarrow H=H^{\prime} \hookrightarrow\left[D\left(\mathscr{A}_{1}^{1 / 2}\right)\right]^{\prime} \subset\left[D\left(\mathscr{A}_{1}\right)\right]^{\prime} .
$$

Define an extension $\tilde{A}_{1} \in \mathcal{L}\left(D\left(\mathscr{A}_{1}^{1 / 2}\right),\left[D\left(\mathscr{A}_{1}^{1 / 2}\right)\right]^{\prime}\right)$ of $\mathscr{A}_{1}$ by

$$
\left\langle\tilde{A}_{1} \varphi, \psi\right\rangle_{\left[D\left(\mathscr{A}_{1}^{1 / 2}\right)\right]^{\prime} \times D\left(\mathscr{A}_{1}^{1 / 2}\right)}=\left\langle\mathscr{A}_{1}^{1 / 2} \varphi, \mathscr{A}_{1}^{1 / 2} \psi\right\rangle_{H}, \forall \varphi, \psi \in D\left(\mathscr{A}_{1}^{1 / 2}\right),
$$

where $\langle\cdot, \cdot\rangle_{H}$ is the inner product on $H^{-1}(\Omega)$. In this way, $i \tilde{A}_{1}$ generates a $C_{0}$-group on $\left[D\left(\mathscr{A}_{1}^{1 / 2}\right)\right]^{\prime}$. Hence (2.6) can be written in $\left[D\left(\mathscr{A}_{1}\right)\right]^{\prime}$ as

$$
\dot{v}=i \tilde{A_{1}} v+B_{1} u,
$$

where $B_{1} \in \mathcal{L}\left(U,\left[D\left(\mathscr{A}_{1}^{1 / 2}\right)\right]^{\prime}\right)$ is given by

$$
B_{1} u=-i \tilde{A_{1}} \Upsilon u, \forall u \in U .
$$

Define $B_{1}^{*} \in \mathcal{L}\left(D\left(\mathscr{A}_{1}^{1 / 2}\right), U\right)$, the adjoint of $B_{1}$, by

$$
\left\langle B_{1} u, f\right\rangle_{\left[D\left(\mathscr{A}_{1}^{1 / 2}\right)\right]^{\prime} \times D\left(\mathscr{A}_{1}^{1 / 2}\right)}=\left\langle u, B_{1}^{*} f\right\rangle_{U}, \forall f \in D\left(\mathscr{A}_{1}^{1 / 2}\right)=L^{2}(\Omega), u \in U .
$$

Then for any $f \in L^{2}(\Omega)$ and $u \in C_{0}^{\infty}(\Gamma)$, we have

$$
\begin{aligned}
& \left\langle B_{1} u, f\right\rangle_{\left[D\left(\mathscr{A}_{1}^{1 / 2}\right)\right]^{\prime} \times D\left(\mathscr{A}_{1}^{1 / 2}\right)} \\
& =\left\langle-i \tilde{A}_{1} \Upsilon u, f\right\rangle_{\left[D\left(\mathscr{A}_{1}^{1 / 2}\right)\right]^{\prime} \times D\left(\mathscr{A}_{1}^{1 / 2}\right)}=-i\left\langle\mathscr{A}_{1}^{1 / 2} \Upsilon u, \mathscr{A}_{1}^{1 / 2} f\right\rangle_{H} \\
& =-i\left\langle\Upsilon u, \mathcal{A}\left(\mathcal{A}^{-1} f\right\rangle_{L^{2}(\Omega)}=\int_{\Omega} \Upsilon u P\left(\overline{i \mathcal{A}^{-1} f}\right) d x\right. \\
& =\int_{\Omega} P(\Upsilon u) \overline{i \mathcal{A}^{-1} f} d x-\int_{\Gamma} \Upsilon u \frac{\partial\left(\overline{i \mathcal{A}^{-1} f}\right)}{\partial \nu_{\mathcal{A}}} d \Gamma+\int_{\Gamma} \frac{\partial(\Upsilon u)}{\partial \nu_{\mathcal{A}}}\left(\overline{i \mathcal{A}^{-1} f}\right) d \Gamma \\
& =\left\langle u,-i \frac{\partial \mathcal{A}^{-1} f}{\partial \nu_{\mathcal{A}}}\right\rangle_{U},
\end{aligned}
$$

where in the last two steps, we used Green's second formula and the definitions of $\Upsilon$ and $\mathcal{A}$. Since $C_{0}^{\infty}(\Gamma)$ is dense in $L^{2}(\Gamma)$, we obtain

$$
B_{1}^{*} f=-i \frac{\partial \mathcal{A}^{-1} f}{\partial \nu_{\mathcal{A}}}, \forall f \in L^{2}(\Omega) .
$$


System (1.1) is then formulated as an abstract form of a first-order system in the state Hilbert space $H$ :

$$
\left\{\begin{aligned}
\dot{v} & =i \tilde{A}_{1} v+B_{1} u, \\
y & =B_{1}^{*} v
\end{aligned}\right.
$$

where $\tilde{A}_{1}, B_{1}$, and $B_{1}^{*}$ are defined by (2.7), (2.9), and (2.10), respectively. The abstract study for system (2.11) can be found in [22].

Next, we formulate the system (1.2) as an abstract second-order system in $\mathcal{H}=$ $H_{0}^{1}(\Omega) \times H$. Let $A$ be the positive self-adjoint operator in $L^{2}(\Omega)$ defined by

$$
\left\{\begin{aligned}
A \varphi=P^{2} \varphi & \\
D(A) & =\left\{\varphi \in L^{2}(\Omega) ; P^{2} \varphi \in L^{2}(\Omega),\left.\varphi\right|_{\Gamma}=\left.P \varphi\right|_{\Gamma}=0\right\} \\
& =\left\{\varphi \in H^{4}(\Omega) ;\left.\varphi\right|_{\Gamma}=\left.P \varphi\right|_{\Gamma}=0\right\} .
\end{aligned}\right.
$$

Just as in [15, it can be shown that

$$
A^{1 / 2}=\mathcal{A},
$$

where $\mathcal{A}$ is given by (1.4), and furthermore, we have the following space identifications with equivalent norms [9, 21]:

$$
\left\{\begin{array}{l}
D\left(A^{\theta}\right)=\left\{\varphi \in H^{4 \theta}(\Omega) ;\left.\varphi\right|_{\Gamma}=0\right\}=H^{4 \theta}(\Omega) \cap H_{0}^{1}(\Omega), 1 / 8<\theta<5 / 8, \\
D\left(A^{\theta}\right)=\left\{\varphi \in H^{4 \theta}(\Omega) ;\left.\varphi\right|_{\Gamma}=\left.P \varphi\right|_{\Gamma}=0\right\}, 5 / 8<\theta \leq 1 .
\end{array}\right.
$$

By (2.12), we have

$$
V \triangleq D\left(A^{3 / 4}\right)=\left\{\varphi \in H^{3}(\Omega) ;\left.\varphi\right|_{\Gamma}=\left.P \varphi\right|_{\Gamma}=0\right\} .
$$

Define an extension operator $\mathscr{A}$ of $A$ to the domain $V$ as

$$
\langle\mathscr{A} \varphi, \psi\rangle_{H}=\left\langle A^{1 / 2} \varphi, A^{1 / 2} \psi\right\rangle_{H}, \forall \varphi, \psi \in V .
$$

Then $\mathscr{A}$ is positive self-adjoint in $H$. In fact, for some constants $C, C^{\prime}>0$, we have

$$
\begin{aligned}
\langle\mathscr{A} \varphi, \varphi\rangle_{H} & =\left\langle A^{1 / 2} \varphi, A^{1 / 2} \varphi\right\rangle_{H}=\left\langle A^{1 / 4} \varphi, A^{1 / 4} \varphi\right\rangle_{L^{2}(\Omega)} \\
& \geq C\|\varphi\|_{L^{2}(\Omega)}^{2} \geq C^{\prime}\left\|A^{-1 / 4} \varphi\right\|_{L^{2}(\Omega)}^{2}=C^{\prime}\|\varphi\|_{H}^{2}, \forall \varphi \in V .
\end{aligned}
$$

We identify $H$ with its dual $H^{\prime}$. Then the following relations hold:

$$
D\left(\mathscr{A}^{1 / 2}\right) \hookrightarrow H=H^{\prime} \hookrightarrow\left[D\left(\mathscr{A}^{1 / 2}\right]^{\prime} .\right.
$$

An extension $\tilde{A} \in \mathcal{L}\left(D\left(\mathscr{A}^{1 / 2}\right),\left[D\left(\mathscr{A}^{1 / 2}\right)\right]^{\prime}\right)$ of $\mathscr{A}$ is defined by

$$
\langle\tilde{A} f, g\rangle_{\left[D\left(\mathscr{A}^{1 / 2}\right)\right]^{\prime} \times D\left(\mathscr{A}^{1 / 2}\right)}=\left\langle\mathscr{A}^{1 / 2} f, \mathscr{A}^{1 / 2} g\right\rangle_{H}, \forall f, g \in D\left(\mathscr{A}^{1 / 2}\right) .
$$

Define the map $G \in \mathcal{L}\left(L^{2}(\Gamma), H^{5 / 2}(\Omega)\right)$ [21, p. 189] so that $G u=\phi$ if and only if

$$
\left\{\begin{array}{l}
P^{2} \phi(x)=0, x \in \Omega \\
\left.\phi(x)\right|_{\Gamma}=0,\left.P \phi(x)\right|_{\Gamma}=u(x) .
\end{array}\right.
$$

By virtue of the operators $\tilde{A}$ and $G$, (1.2) can be written in $\left[D\left(\mathscr{A}^{1 / 2}\right)\right]^{\prime}$ as

$$
\ddot{w}+\tilde{A} w=B u,
$$


where $B \in \mathcal{L}\left(U,\left[D\left(\mathscr{A}^{1 / 2}\right)\right]^{\prime}\right)$ is given by

$$
B u=\tilde{A} G u, \forall u \in U \text {. }
$$

Define $B^{*} \in \mathcal{L}\left(D\left(\mathscr{A}^{1 / 2}\right), U\right)$ by

$$
\langle B u, f\rangle_{\left[D\left(A^{1 / 2}\right)\right]^{\prime} \times D\left(A^{1 / 2}\right)}=\left\langle u, B^{*} f\right\rangle_{U}, \forall f \in D\left(\mathscr{A}^{1 / 2}\right)=H_{0}^{1}(\Omega), u \in U .
$$

Then for any $f \in D\left(\mathscr{A}^{1 / 2}\right)$ and $u \in C_{0}^{\infty}(\Gamma)$, we have

$$
\begin{aligned}
& \langle B u, f\rangle_{\left[D\left(A^{1 / 2}\right)\right]^{\prime} \times D\left(A^{1 / 2}\right)} \\
& =\langle\tilde{A} G u, f\rangle_{\left[D\left(A^{1 / 2}\right)\right]^{\prime} \times D\left(A^{1 / 2}\right)}=\left\langle\mathscr{A}^{1 / 2} G u, \mathscr{A}^{1 / 2} f\right\rangle_{H} \\
& =\left\langle\mathscr{A} G u, \mathcal{A}\left(\mathcal{A}^{-1} f\right)\right\rangle_{H}=\left\langle A^{-1 / 4} A G u, A^{-1 / 4} \mathcal{A}\left(\mathcal{A}^{-1} f\right)\right\rangle_{L^{2}(\Omega)} \\
& =\left\langle\mathcal{A} G u, \mathcal{A}\left(\mathcal{A}^{-1} f\right)\right\rangle_{L^{2}(\Omega)} \\
& =\int_{\Omega} P^{2}(G u) \overline{\mathcal{A}^{-1} f} d x+\int_{\Gamma} P(G u) \frac{\partial \overline{\mathcal{A}^{-1} f}}{\partial \nu_{\mathcal{A}}} d \Gamma+\int_{\Gamma}\left(\mathcal{A}^{-1} f\right) \frac{\partial \overline{P(G u)}}{\partial \nu_{\mathcal{A}}} d \Gamma \\
& =\left\langle u, \frac{\partial \mathcal{A}^{-1} f}{\partial \nu_{\mathcal{A}}}\right\rangle_{U},
\end{aligned}
$$

where we used Green's second formula and the definitions of $G$ and $\mathcal{A}$. Since $C_{0}^{\infty}(\Gamma)$ is dense in $L^{2}(\Gamma)$, we actually obtain

$$
B^{*} f=\left.\frac{\partial \mathcal{A}^{-1} f}{\partial \nu_{\mathcal{A}}}\right|_{\Gamma}, \forall f \in D\left(\mathscr{A}^{1 / 2}\right)=H_{0}^{1}(\Omega)
$$

Finally, we cast the open-loop system (1.2) into the abstract form of a second-order collocated system in $\mathcal{H}$,

$$
\left\{\begin{array}{l}
\ddot{w}+\tilde{A} w=B u, \\
y=B^{*} \dot{w},
\end{array}\right.
$$

where $\tilde{A}, B$, and $B^{*}$ are defined by (2.14), (2.17), and (2.18), respectively. System (2.19) was well studied in [2, 10].

3. Proof of Theorem 1.1. For brevity in notation, we shall denote by $C_{T}$, in subsequent sections, a positive constant depending only on the time $T$ that may have different values in different contexts. First, we show that $B_{1}$ is admissible for $e^{i \mathscr{A}_{1} t}$, the $C_{0}$-group generated by $i \mathscr{A}_{1}$ on $H$. Since the system (1.1) is collocated, $B_{1}$ is admissible for $e^{i \mathscr{A}_{1} t}$ if and only if $B_{1}^{*}$ is admissible for $e^{-i \mathscr{A}_{1}^{*} t}=e^{i \mathscr{A}_{1} t}$ (see [29]). Therefore, we need to show the estimate

$$
\int_{0}^{T}\left\|B_{1}^{*}\left(e^{i \mathscr{A}_{1} t} v_{0}\right)\right\|_{L^{2}(\Gamma)}^{2} d t \leq C_{T}\left\|v_{0}\right\|_{H^{-1}(\Omega)}^{2}, \forall v_{0} \in D\left(\mathscr{A}_{1}\right)=H_{0}^{1}(\Omega) .
$$


Set $z=\mathscr{A}_{1}^{-1} v$. Instead of (2.11), we consider the following $z$ equation in the space $H_{0}^{1}(\Omega)$ that is derived from (2.9), (2.10), and (2.11):

$$
\left\{\begin{array}{lll}
z_{t}(x, t)=i P z(x, t)-i(\Upsilon u(\cdot, t))(x), & & (x, t) \in \Omega \times(0, T], \\
z(x, 0)=z_{0}(x), & & x \in \Omega, \\
z(x, t)=0, & & (x, t) \in \Gamma \times[0, T], \\
y(x, t)=-i \frac{\partial z(x, t)}{\partial \nu_{\mathcal{A}}}, & & (x, t) \in \Gamma \times[0, T] .
\end{array}\right.
$$

Let $f(x, t)=-i(\Upsilon u(\cdot, t))(x)$. By the definition of the Dirichlet map $\Upsilon$, we have

$$
\int_{0}^{T}\|f(\cdot, t)\|_{L^{2}(\Omega)}^{2} d t \leq C_{T} \int_{0}^{T}\|u(\cdot, t)\|_{L^{2}(\Gamma)}^{2} d t .
$$

By Lemma 4.1 of [15], there exists a $C^{2}$ vector field $N$ on $\bar{\Omega}$ such that

$$
N(x)=\mu(x), x \in \Gamma ;|N(x)|_{g} \leq 1, \quad x \in \Omega .
$$

Multiply both sides of the first equation in (3.2) by $N(\bar{z})$ and integrate over $\Omega$, to give

$$
\begin{aligned}
0= & \int_{\Omega} z_{t} N(\bar{z}) d x-i \int_{\Omega} P z N(\bar{z}) d x-\int_{\Omega} f N(\bar{z}) d x \\
= & \int_{\Omega} z_{t} N(\bar{z}) d x+i \int_{\Omega}\left(\Delta_{g} z-(D q) z\right) N(\bar{z}) d x-\int_{\Omega} f N(\bar{z}) d x \\
= & \int_{\Omega} z_{t} N(\bar{z}) d x+i \int_{\Gamma}\left|\frac{\partial z}{\partial \mu}\right|^{2} d \Gamma-i \int_{\Omega}\left\langle\nabla_{g} z, \nabla_{g}(N(\bar{z}))\right\rangle_{g} d x \\
& -i \int_{\Omega}(D q) z N(\bar{z}) d x-\int_{\Omega} f N(\bar{z}) d x
\end{aligned}
$$

It then follows that

$$
\begin{aligned}
\int_{\Gamma}\left|\frac{\partial z}{\partial \mu}\right|^{2} d \Gamma & =\operatorname{Re} \int_{\Omega}\left\langle\nabla_{g} z, \nabla_{g}(N(\bar{z}))\right\rangle_{g} d x \\
& -\operatorname{Im} \int_{\Omega} z_{t} N(\bar{z}) d x+\operatorname{Re} \int_{\Omega}(D q) z N(\bar{z}) d x+\operatorname{Im} \int_{\Omega} f N(\bar{z}) d x .
\end{aligned}
$$

By Lemma 2.2, one has

$$
\operatorname{Re}\left\langle\nabla_{g} z, \nabla_{g}(N(\bar{z}))\right\rangle_{g}=\operatorname{Re} D N\left(\nabla_{g} z, \nabla_{g} \bar{z}\right)+\frac{1}{2} \operatorname{div}_{g}\left(\left|\nabla_{g} z\right|_{g}^{2} N\right)-\frac{1}{2}\left|\nabla_{g} z\right|_{g}^{2} \operatorname{div}_{g} N .
$$

Substitute (3.6) into (3.5), to obtain

$$
\begin{aligned}
\int_{\Gamma}\left|\frac{\partial z}{\partial \mu}\right|^{2} d \Gamma= & \operatorname{Re} \int_{\Omega} D N\left(\nabla_{g} z, \nabla_{g} \bar{z}\right) d x \\
& +\frac{1}{2} \int_{\Gamma}\left|\nabla_{g} z\right|_{g}^{2} d \Gamma-\frac{1}{2} \int_{\Omega}\left|\nabla_{g} z\right|_{g}^{2} \operatorname{div}_{g} N d x \\
& -\operatorname{Im} \int_{\Omega} z_{t} N(\bar{z}) d x+\operatorname{Re} \int_{\Omega}(D q) z N(\bar{z}) d x+\operatorname{Im} \int_{\Omega} f N(\bar{z}) d x
\end{aligned}
$$


Notice that for any $x \in \Gamma$, it has

$$
\nabla_{g} z(x)=\left\langle\nabla_{g} z(x), \mu(x)\right\rangle_{g} \mu(x)+Y(x)=\frac{\partial z(x)}{\partial \mu} \mu(x)+Y(x)
$$

for some $Y(x) \in \mathbb{R}_{x}^{n}$ with $\langle Y(x), \mu(x)\rangle_{g}=0$, which implies that $Y(x) \cdot \nu(x)=0$. This fact together with the boundary condtion $\left.z\right|_{\Gamma}=0$ gives

$$
\begin{aligned}
\left|\nabla_{g} z(x)\right|_{g}^{2} & =\left\langle\nabla_{g} z(x), \nabla_{g} \bar{z}(x)\right\rangle_{g} \\
& =\nabla_{g} z(x)(\bar{z}(x))=\left|\frac{\partial z(x)}{\partial \mu}\right|^{2}=\frac{1}{\left|\nu_{\mathcal{A}}(x)\right|_{g}^{2}}\left|\frac{\partial z(x)}{\partial \nu_{\mathcal{A}}}\right|^{2}, \forall x \in \Gamma,
\end{aligned}
$$

where we used the fact $\mu=\frac{\nu_{\mathcal{A}}}{\left|\nu_{\mathcal{A}}\right|_{g}}$. In addition, by (1.3), (1.5), and (2.2), we have

$$
\min _{x \in \Gamma} \frac{1}{\left|\nu_{\mathcal{A}}(x)\right|_{g}^{2}} \geq \frac{1}{b}
$$

By (3.8) and (3.9), making use of the Cauchy-Schwartz inequality and Lemma 2.3, we deduce, from (3.7), that

$$
\begin{aligned}
& \int_{\Gamma}\left|\frac{\partial z}{\partial \nu_{\mathcal{A}}}\right|^{2} d \Gamma \leq b \int_{\Gamma}\left|\frac{\partial z}{\partial \mu}\right|^{2} d \Gamma \\
& =2 b \operatorname{Re} \int_{\Omega} D N\left(\nabla_{g} z, \nabla_{g} \bar{z}\right) d x-b \int_{\Omega}\left|\nabla_{g} z\right|_{g}^{2} \operatorname{div}_{g} N d x-2 b \operatorname{Im} \int_{\Omega} z_{t} N(\bar{z}) d x \\
& \quad+2 b \operatorname{Re} \int_{\Omega}(D q) z N(\bar{z}) d x+2 b \operatorname{Im} \int_{\Omega} f N(\bar{z}) d x \\
& \leq C\left(\int_{\Omega}\left|\nabla_{g} z\right|_{g}^{2} d x+\int_{\Omega}|f|^{2} d x\right)-2 b \operatorname{Im} \int_{\Omega} z_{t} N(\bar{z}) d x .
\end{aligned}
$$

Now we compute the last term in (3.10). By the divergence formula and (3.2), it follows that

$$
\begin{aligned}
& \operatorname{div}_{g}\left(z_{t} \bar{z} N\right)=z_{t} \bar{z} \operatorname{div}_{g} N+N\left(z_{t}\right) \bar{z}+N(\bar{z}) z_{t} \\
& =(i P z+f) \bar{z} \operatorname{div}_{g} N+\frac{d}{d t}(\bar{z} N(z))-\bar{z}_{t} N(z)+N(\bar{z}) z_{t} \\
& =(i P z+f) \bar{z} \operatorname{div}_{g} N+\frac{d}{d t}(\bar{z} N(z))+2 i \operatorname{Im} z_{t} N(\bar{z})
\end{aligned}
$$

Integrate the above equality over $\Omega$, to give

$$
\begin{aligned}
& 2 i \operatorname{Im} \int_{\Omega} z_{t} N(\bar{z}) d x=\int_{\Omega}\left[i\left(\Delta_{g}-D q\right) z-f\right] \bar{z} \operatorname{div}_{g} N d x-\frac{d}{d t} \int_{\Omega} \bar{z} N(z) d x \\
& =-i \int_{\Omega}\left\langle\nabla_{g} z, \nabla_{g}\left(\bar{z} \operatorname{div}_{g} N\right)\right\rangle_{g} d x-i \int_{\Omega} D q(z) \bar{z} \operatorname{div}_{g} N d x-\int_{\Omega} f \bar{z} \operatorname{div}_{g} N d x \\
& \quad-\frac{d}{d t} \int_{\Omega} \bar{z} N(z) d x .
\end{aligned}
$$


Therefore,

$$
\begin{aligned}
& -2 b \operatorname{Im} \int_{0}^{T} \int_{\Omega} z_{t} N(\bar{z}) d x d t \\
& =\int_{0}^{T} \int_{\Omega}\left\langle\nabla_{g} z, \nabla_{g}\left(\bar{z} \operatorname{div}_{g}(N)\right)\right\rangle_{g} d x d t+\int_{0}^{T} \int_{\Omega} D q(z) \bar{z} \operatorname{div}_{g}(N) d x d t \\
& +i \int_{0}^{T} \int_{\Omega} f \bar{z} \operatorname{div}_{g}(N) d x d t+\left.i \int_{\Omega} \bar{z} N(z) d x\right|_{0} ^{T} .
\end{aligned}
$$

Using (3.11) and integrating both sides of (3.10) over $[0, T]$, we obtain

$$
\begin{aligned}
\int_{0}^{T} \int_{\Gamma}\left|\frac{\partial z}{\partial \nu_{\mathcal{A}}}\right|^{2} d \Gamma d t & \leq C_{T}\left(\|z\|_{L^{2}\left(0, T ; H^{1}(\Omega)\right)}^{2}\right. \\
& \left.+\|f\|_{L^{2}(\Omega \times(0, T))}^{2}+\|z\|_{L^{\infty}\left(0, T ; H^{1}(\Omega)\right)}^{2}\right) .
\end{aligned}
$$

Let $f=-i \Upsilon u=0$ in (3.2). For any $z_{0} \in D\left(\mathscr{A}_{1}\right)=H_{0}^{1}(\Omega)$, it has

$$
e^{i \mathscr{A}_{1} t} z_{0} \in C\left([0, T] ; D\left(\mathscr{A}_{1}\right)\right) \cap C^{1}((0, T] ; H),
$$

and hence, by (3.12), we have

$$
\int_{0}^{T} \int_{\Gamma}\left|\frac{\partial\left(e^{i \mathscr{A}_{1} t} z_{0}\right)}{\partial \nu_{\mathcal{A}}}\right|^{2} d \Gamma d t \leq C_{T}\left\|z_{0}\right\|_{D\left(\mathscr{A}_{1}\right)}^{2}, \forall z_{0} \in D\left(\mathscr{A}_{1}\right) .
$$

This implies

$$
\int_{0}^{T} \int_{\Gamma}\left|\frac{\partial\left(e^{i \mathscr{A}_{1} t} \mathscr{A}_{1}^{-1} v_{0}\right)}{\partial \nu_{\mathcal{A}}}\right|^{2} d \Gamma d t \leq C_{T}\left\|v_{0}\right\|_{H^{-1}(\Omega)}^{2}, \forall v_{0} \in D\left(\mathscr{A}_{1}\right) .
$$

By the definition of $B_{1}^{*},(3.13)$ is just (3.1). Therefore, $B_{1}$ is admissible.

Next, we show the boundedness of input-output map for the system (3.2), that is, for some (and hence for all) $T>0$, the solution to (3.2) with $z_{0}=0$ satisfies

$$
\int_{0}^{T} \int_{\Gamma}\left|\frac{\partial z(x, t)}{\partial \nu_{\mathcal{A}}}\right|^{2} d \Gamma d t \leq C_{T} \int_{0}^{T} \int_{\Gamma}|u(x, t)|^{2} d \Gamma d t, \forall u \in L^{2}\left(0, T ; L^{2}(\Gamma)\right) .
$$

Noticing that the solution to (3.2) with $z_{0}=0$ is given by

$$
z(x, t)=\int_{0}^{t}\left[e^{i \tilde{A_{1}}(t-s)} f(\cdot, s)\right](x) d s=-i \int_{0}^{t}\left[e^{i \tilde{A_{1}}(t-s)} \Upsilon u(\cdot, s)\right](x) d s,
$$

and the admissibility just verified, we have [30]

$$
\begin{aligned}
\tilde{A}_{1} z(x, t) & =-i \int_{0}^{t}\left[e^{i \tilde{A_{1}}(t-s)} \tilde{A_{1}} \Upsilon u(\cdot, s)\right](x) d s \\
& =\int_{0}^{t}\left[e^{i \tilde{A_{1}}(t-s)} B_{1} u(\cdot, s)\right](x) d s \in C([0, T] ; H) .
\end{aligned}
$$

It then follows that

$$
z \in C\left([0, T] ; H_{0}^{1}(\Omega)\right) .
$$

This together with (3.3) and (3.12) shows that (3.15) is valid. The proof is complete.

REMARK 3.1. From Proposition 4.1 of [23, (3.15) implies the admissibility of $B$ (so is for $\left.B^{*}\right)$, but we do not use this fact here. 
4. Proof of Theorem 1.2. By definition of the well-posedness (see [6]), Theorem 1.2 is equivalent to saying that $B$ is admissible (so is $B^{*}$ ), and the solution to (1.2) with zero initial data satisfies

$$
\|y\|_{L^{2}\left(0, T ; L^{2}(\Gamma)\right)}^{2} \leq C_{T}\|u\|_{L^{2}\left(0, T ; L^{2}(\Gamma)\right)}^{2}, \forall u \in L^{2}\left(0, T ; L^{2}(\Gamma)\right) .
$$

Introduce the following transform

$$
z(t)=\mathcal{A}^{-1} w(t) \in C(0, T ; V) .
$$

Instead of (1.2), we consider the following system in $V \times H_{0}^{1}(\Omega)$ :

$$
\begin{cases}z_{t t}(x, t)+P^{2} z(x, t)=\Upsilon u(x, t), & (x, t) \in \Omega \times(0, T] \triangleq Q, \\ z(x, 0)=0, z_{t}(x, 0)=0, & x \in \Omega, \\ z(x, t)=0, P z(x, t)=0, & (x, t) \in \Gamma \times(0, T] \triangleq \Sigma, \\ y(x, t)=\frac{\partial z_{t}}{\partial \nu_{\mathcal{A}}}(x, t), & (x, t) \in \Sigma,\end{cases}
$$

where the operator $\Upsilon$ is defined by (2.5), and the validity of the first equation in (4.3) is attributed to the fact

$$
\mathcal{A}^{-1} \tilde{A} G u=\tilde{A}^{1 / 2} G u=\mathcal{A} G u=\Upsilon u, \forall u \in L^{2}(\Gamma) .
$$

Therefore, Theorem 1.2 is valid if and only if $B$ is admissible, and for some (and hence for all) $T>0$, there exists a $C_{T}>0$ such that the solution to (4.3) satisfies

$$
\int_{\Sigma}\left|\frac{\partial z_{t}}{\partial \nu_{\mathcal{A}}}(x, t)\right|^{2} d \Sigma \leq C_{T} \int_{\Sigma}|u(x, t)|^{2} d \Sigma .
$$

Step 1 (Energy identity). Let $N$ be the $C^{2}$ vector field on $\bar{\Omega}$ satisfying (3.4). Multiply the both sides of (4.3) by $N(P \bar{z})$, to give

$$
\int_{Q} z_{t t} N(P \bar{z}) d Q+\int_{Q} P^{2} z N(P \bar{z}) d Q-\int_{Q} \Upsilon u N(P \bar{z}) d Q=0 .
$$

Compute the first term on the left-hand side of (4.5) to yield

$$
\begin{aligned}
& \int_{Q} z_{t t} N(P \bar{z}) d Q=\left.\int_{\Omega} z N(P \bar{z}) d x\right|_{0} ^{T}-\int_{Q} z_{t} N\left(P \overline{z_{t}}\right) d Q \\
& =\left.\int_{\Omega} z N(P \bar{z}) d x\right|_{0} ^{T}-\int_{Q}\left[N\left(z_{t} P \overline{z_{t}}\right)-N\left(z_{t}\right) P \overline{z_{t}}\right] d Q \\
& =\left.\int_{\Omega} z N(P \bar{z}) d x\right|_{0} ^{T}-\int_{\Sigma} z_{t}\left(P \overline{z_{t}}\right)\langle N, \mu\rangle_{g} d \Sigma+\int_{Q} z_{t}\left(P \overline{z_{t}}\right) \operatorname{div}_{g} N d Q+\int_{Q} N\left(z_{t}\right) P \overline{z_{t}} d Q \\
& =\left.\int_{\Omega} z N(P \bar{z}) d x\right|_{0} ^{T}+\int_{Q} z_{t}\left(P \overline{z_{t}}\right) \operatorname{div}_{g} N d Q+\int_{Q} N\left(z_{t}\right) P \overline{z_{t}} d Q
\end{aligned}
$$


Since

$$
\begin{aligned}
& \operatorname{Re} \int_{Q} z_{t}\left(P \overline{z_{t}}\right) \operatorname{div}_{g} N d Q=-\operatorname{Re} \int_{Q} z_{t} \operatorname{div}_{g} N \Delta_{g} \overline{z_{t}} d Q+\operatorname{Re} \int_{Q} z_{t} D q\left(\overline{z_{t}}\right) d Q \\
& =-\operatorname{Re} \int_{\Sigma} z_{t} \operatorname{div}_{g} N \frac{\partial \overline{z_{t}}}{\partial \mu} d \Sigma+\operatorname{Re} \int_{Q}\left\langle\nabla_{g}\left(z_{t} \operatorname{div}_{g} N\right), \nabla_{g} \overline{z_{t}}\right\rangle_{g} d Q+\operatorname{Re} \int_{Q} z_{t} D q\left(\overline{z_{t}}\right) d Q \\
& =\int_{Q} \operatorname{div}_{g} N\left|\nabla_{g} z_{t}\right|_{g}^{2} d Q+\operatorname{Re} \int_{Q} z_{t} \nabla_{g}\left(\operatorname{div}_{g} N\right)\left(\overline{z_{t}}\right) d Q+\operatorname{Re} \int_{Q} z_{t} D q\left(\overline{z_{t}}\right) d Q \\
& =\int_{Q} \operatorname{div}_{g} N\left|\nabla_{g} z_{t}\right|_{g}^{2} d Q+\frac{1}{2} \int_{Q} \nabla_{g}\left(\operatorname{div}_{g} N\right)\left(\left|z_{t}\right|^{2}\right) d Q+\frac{1}{2} \int_{Q} D q\left(\left|z_{t}\right|^{2}\right) d Q,
\end{aligned}
$$

and

$$
\begin{aligned}
\operatorname{Re} \int_{Q} N\left(z_{t}\right) P \overline{z_{t}} d Q=-\operatorname{Re} \int_{Q} N\left(z_{t}\right) \Delta_{g} \overline{z_{t}} d Q+\operatorname{Re} \int_{Q} N\left(z_{t}\right) D q\left(\overline{z_{t}}\right) d Q \\
=-\operatorname{Re} \int_{\Sigma} N\left(z_{t}\right) \frac{\partial \overline{z_{t}}}{\partial \mu} d \Sigma+\operatorname{Re} \int_{Q}\left\langle\nabla_{g} \overline{z_{t}}, \nabla_{g}\left(N\left(z_{t}\right)\right)\right\rangle_{g} d Q+\operatorname{Re} \int_{Q} N\left(z_{t}\right) D q\left(\overline{z_{t}}\right) d Q \\
=-\int_{\Sigma}\left|\frac{\partial z_{t}}{\partial \mu}\right|^{2} d \Sigma+\operatorname{Re} \int_{Q} D N\left(\nabla_{g} z_{t}, \nabla_{g} \overline{z_{t}}\right) d Q+\frac{1}{2} \int_{Q} \operatorname{div}_{g}\left(\left|\nabla_{g} z_{t}\right|_{g}^{2} N\right) d Q \\
-\frac{1}{2} \int_{Q}\left|\nabla_{g} z_{t}\right|_{g}^{2} \operatorname{div}_{g} N d Q+\operatorname{Re} \int_{Q} N\left(z_{t}\right) D q\left(\overline{z_{t}}\right) d Q \\
=-\frac{1}{2} \int_{\Sigma}\left|\frac{\partial z_{t}}{\partial \mu}\right|^{2} d \Sigma+\operatorname{Re} \int_{Q} D N\left(\nabla_{g} z_{t}, \nabla_{g} \overline{z_{t}}\right) d Q-\frac{1}{2} \int_{Q}\left|\nabla_{g} z_{t}\right|_{g}^{2} \operatorname{div}{ }_{g} N d Q \\
\quad+\operatorname{Re} \int_{Q} N\left(z_{t}\right) D q\left(\overline{z_{t}}\right) d Q,
\end{aligned}
$$

where the formulae (3.6) and (3.8) were used for the computation of the above identity, it follows that

$$
\begin{aligned}
& \operatorname{Re} \int_{Q} z_{t t} N(P \bar{z}) d Q=\left.\operatorname{Re} \int_{\Omega} z N(P \bar{z}) d x\right|_{0} ^{T}+\frac{1}{2} \int_{Q} \operatorname{div}_{g} N\left|\nabla_{g} z_{t}\right|_{g}^{2} d Q \\
& \quad+\frac{1}{2} \int_{Q} \nabla_{g}\left(\operatorname{div}_{g} N\right)\left(\left|z_{t}\right|^{2}\right) d Q+\frac{1}{2} \int_{Q} D q\left(\left|z_{t}\right|^{2}\right) d Q-\frac{1}{2} \int_{\Sigma}\left|\frac{\partial z_{t}}{\partial \mu}\right|^{2} d \Sigma \\
& \quad+\operatorname{Re} \int_{Q} D N\left(\nabla_{g} z_{t}, \nabla_{g} \overline{z_{t}}\right) d Q+\operatorname{Re} \int_{Q} N\left(z_{t}\right) D q\left(\overline{z_{t}}\right) d Q
\end{aligned}
$$


By Green's first formula, (3.6) and (3.8), the second term on the left-hand side of (4.5) is computed as

$$
\begin{aligned}
& \operatorname{Re} \int_{Q} P^{2} z N(P \bar{z}) d Q=\operatorname{Re} \int_{Q}\left\langle\nabla_{g}(P z), \nabla_{g}(N(P \bar{z}))\right\rangle_{g} d Q \\
& -\operatorname{Re} \int_{\Sigma} \frac{\partial P z}{\partial \mu} N(P \bar{z}) d \Sigma=\operatorname{Re} \int_{Q} D N\left(\nabla_{g}(P z), \nabla_{g}(P \bar{z})\right) d Q \\
& +\frac{1}{2} \int_{Q} \operatorname{div}_{g}\left(\left|\nabla_{g}(P z)\right|_{g}^{2} N\right) d Q-\frac{1}{2} \int_{Q}\left|\nabla_{g}(P z)\right|_{g}^{2} \operatorname{div}_{g} N d Q \\
& -\int_{\Sigma}\left|\frac{\partial P z}{\partial \mu}\right|^{2} d \Sigma=\operatorname{Re} \int_{Q} D N\left(\nabla_{g}(P z), \nabla_{g}(P \bar{z})\right) d Q \\
& -\frac{1}{2} \int_{Q}\left|\nabla_{g}(P z)\right|_{g}^{2} \operatorname{div}_{g} N d Q-\frac{1}{2} \int_{\Sigma}\left|\frac{\partial P z}{\partial \mu}\right|^{2} d \Sigma .
\end{aligned}
$$

Combine (4.5), (4.6), and (4.7), to obtain

$$
\frac{1}{2} \int_{\Sigma}\left(\left|\frac{\partial z_{t}}{\partial \mu}\right|^{2}+\left|\frac{\partial P z}{\partial \mu}\right|^{2}\right) d \Sigma=R_{1}+R_{2}+b_{T}
$$

where

$$
\begin{gathered}
R_{1}=\operatorname{Re} \int_{Q} D N\left(\nabla_{g}(P z), \nabla_{g}(P \bar{z})\right) d Q+\operatorname{Re} \int_{Q} D N\left(\nabla_{g} z_{t}, \nabla_{g} \overline{z_{t}}\right) d Q \\
+\frac{1}{2} \int_{Q} D q\left(\left|z_{t}\right|^{2}\right) d Q+\frac{1}{2} \int_{Q} \operatorname{div}_{g} N\left(\left|\nabla_{g} z_{t}\right|_{g}^{2}-\left|\nabla_{g}(P z)\right|_{g}^{2}\right) d Q \\
+\frac{1}{2} \int_{Q} \nabla_{g}\left(\operatorname{div}_{g} N\right)\left(\left|z_{t}\right|^{2}\right) d Q+\operatorname{Re} \int_{Q} N\left(z_{t}\right) D q\left(\overline{z_{t}}\right) d Q, \\
R_{2}=-\int_{Q} \Upsilon u N(P \bar{z}) d Q, \\
b_{T}=\left.\operatorname{Re} \int_{\Omega} z N(P \bar{z}) d x\right|_{0} ^{T} .
\end{gathered}
$$

Step 2 (Estimate of $R_{1}$ ). It is easy to show

$$
\left\{\begin{array}{l}
z_{t t}+\mathscr{A} z=0, \\
z(0)=z_{0}, z_{t}(0)=z_{1}, \\
y=B^{*} \mathcal{A} z_{t},
\end{array}\right.
$$

where $\mathscr{A}$ and $\mathcal{A}$ are given by (2.13) and (1.4), respectively, which is the dual system of (2.16), associates with a $C_{0}$-group solution in the space $V \times H_{0}^{1}(\Omega)$. That is to say, for any $\left(z_{0}, z_{1}\right) \in V \times H_{0}^{1}(\Omega)$, the corresponding solution to (4.12) satisfies $\left(z, z_{t}\right) \in V \times H_{0}^{1}(\Omega)$, and depends continuously on $\left(z_{0}, z_{1}\right)$. By this fact, letting $\Upsilon u=0$ in (4.8) and using (3.8) and (3.9), we obtain from (4.8) that

$$
\int_{\Sigma}\left|\frac{\partial z_{t}}{\partial \nu_{\mathcal{A}}}\right|^{2} d \Sigma \leq C_{T}\left\|\left(z_{0}, z_{1}\right)\right\|_{V \times H_{0}^{1}(\Omega)}^{2}
$$


which is equivalent to saying that for any initial data $\left(w_{0}, w_{1}\right) \in H_{0}^{1}(\Omega) \times H$, the solution to the system (1.2) with $u=0$ satisfies

$$
\int_{\Sigma}\left|\frac{\partial \mathcal{A}^{-1} w_{t}}{\partial \nu_{\mathcal{A}}}\right|^{2} d \Sigma \leq C_{T}\left\|\left(w_{0}, w_{1}\right)\right\|_{H_{0}^{1}(\Omega) \times H}^{2} .
$$

This shows that $B^{*}$ is admissible; so is $B$ [29]. In other words,

$$
u \mapsto\left\{w, w_{t}\right\} \text { is continuous from } L^{2}(\Sigma) \rightarrow C\left([0, T] ; H_{0}^{1}(\Omega) \times H\right) .
$$

By (4.15), we have

$$
\left(z, z_{t}\right) \in C\left([0, T] ; V \times H_{0}^{1}(\Omega)\right) \text { continuously in } u \in L^{2}(\Sigma) .
$$

Hence

$$
R_{1} \leq C_{T}\|u\|_{L^{2}(\Sigma)}^{2}, \forall u \in L^{2}(\Sigma),
$$

where we used Lemma 2.3 .

Step 3 (Estimate of $R_{2}$ and $b_{T}$ ). From (4.10) and (4.11), by virtue of (4.16), we readily obtain

$$
R_{2}+b_{T} \leq C_{T}\|u\|_{L^{2}(\Sigma)}^{2}, \forall u \in L^{2}(\Sigma) .
$$

Step 4 (Final estimate). Combining (4.17), (4.18), and (4.8), and using (3.8) and (3.9), we finally obtain

$$
\int_{\Sigma}\left|\frac{\partial z_{t}}{\partial \nu_{\mathcal{A}}}(x, t)\right|^{2} d \Sigma+\int_{\Sigma}\left|\frac{\partial P z}{\partial \nu_{\mathcal{A}}}(x, t)\right|^{2} d \Sigma \leq C_{T} \int_{\Sigma}|u(x, t)|^{2} d \Sigma .
$$

This concludes (4.4). This together with (4.15) completes the proof of Theorem 1.2.

REMARK 4.1. It was realized in Corollary A.2 of [22] that (4.1), the boundedness of the input-output map, implies the admissibility of $B$ (so is for $B^{*}$ ), but the proof there is incorrect. A simple proof was given in [4]. But here we do not need this fact.

5. Proofs of Theorems 1.4 and 1.5. It is known from the Appendix of [10] that the transfer functions of the systems (2.11) and (2.19) are, respectively,

$$
H_{1}(\lambda)=B_{1}^{*}\left(\lambda-i \tilde{A}_{1}\right)^{-1} B_{1}
$$

and

$$
H(\lambda)=\lambda B^{*}\left(\lambda^{2}+\tilde{A}\right)^{-1} B,
$$

where $\tilde{A}_{1}, B_{1}$, and $B_{1}^{*}$ are given by (2.7), (2.9), and (2.10); $\tilde{A}, B$, and $B^{*}$ are given by (2.14), (2.17), and (2.18), respectively. Moreover, from the well-posedness claimed by Theorems 1.1 and 1.2 , it follows that there exist constants $M, \alpha, \beta>0$ such that 8

$$
\sup _{\operatorname{Re} \lambda \geq \alpha}\left\|H_{1}(\lambda)\right\|_{\mathcal{L}(U)}<M
$$

and

$$
\sup _{\operatorname{Re} \lambda \geq \beta}\|H(\lambda)\|_{\mathcal{L}(U)}<M
$$


Proposition 5.1. Theorem 1.4 is valid if for any $u \in C_{0}^{\infty}(\Gamma)$ and $\varepsilon>0$, the solution $v_{\varepsilon}$ to the following equation

$$
\begin{cases}\varepsilon^{-1} v_{\varepsilon}(x)-i P v_{\varepsilon}(x)=0, & x \in \Omega, \\ v_{\varepsilon}(x)=u(x), & x \in \Gamma,\end{cases}
$$

satisfies

$$
\lim _{\varepsilon \rightarrow 0+} \int_{\Gamma}\left|\varepsilon \frac{\partial v_{\varepsilon}}{\partial \nu_{\mathcal{A}}}\right|^{2} d \Gamma=0
$$

Proof. We need only show that $H_{1}(\lambda) u$ converges to zero in the strong topology of $U$ along the positive axis (see 32]), that is,

$$
\lim _{\lambda \in \mathbb{R}, \lambda \rightarrow+\infty} H_{1}(\lambda) u=0, \forall u \in L^{2}(\Gamma) .
$$

Due to (5.3) and a density argument, it suffices to show that (5.7) is satisfied for all $u \in C_{0}^{\infty}(\Gamma)$. To this end, let $u \in C_{0}^{\infty}(\Gamma)$. Denote by

$$
v_{\lambda}=\left(\lambda-i \tilde{A}_{1}\right)^{-1} B_{1} u \text {. }
$$

Then along the line from (2.5) to (2.9), we conclude that $v_{\lambda}$ satisfies

$$
\begin{cases}\lambda v_{\lambda}(x)-i P v_{\lambda}(x)=0, & x \in \Omega \\ v_{\lambda}(x)=u(x), & x \in \Gamma .\end{cases}
$$

It follows from (2.10), (5.8), and (5.1) that

$$
\left(H_{1}(\lambda) u\right)(x)=-i \frac{\partial\left(\mathcal{A}^{-1} v_{\lambda}\right)}{\partial \nu_{\mathcal{A}}}(x), \forall x \in \Gamma .
$$

Since $u \in C_{0}^{\infty}(\Gamma)$, there exists a unique classical solution to (5.9). Let $\tilde{v} \in C^{\infty}(\Omega)$ be the unique solution to the boundary value problem

$$
\begin{cases}P \tilde{v}(x)=0, & x \in \Omega \\ \tilde{v}(x)=u(x), & x \in \Gamma .\end{cases}
$$

Then (5.9) can be written as

$$
\begin{cases}i \lambda v_{\lambda}(x)-P\left(v_{\lambda}(x)-\tilde{v}(x)\right)=0, & x \in \Omega, \\ v_{\lambda}(x)-\tilde{v}(x)=0, & x \in \Gamma,\end{cases}
$$

or equivalently,

$$
i \lambda \mathcal{A}^{-1} v_{\lambda}=v_{\lambda}-\tilde{v}
$$

Hence, (5.10) becomes

$$
\left(H_{1}(\lambda) u\right)(x)=-\frac{1}{\lambda} \frac{\partial v_{\lambda}}{\partial \nu_{\mathcal{A}}}(x)+\frac{1}{\lambda} \frac{\partial \tilde{v}}{\partial \nu_{\mathcal{A}}}(x), \forall x \in \Gamma .
$$

Letting $v_{\varepsilon}=v_{\lambda}$ with $\varepsilon=\frac{1}{\lambda}$, we obtain the required result. 
Proof of Theorem 1.4. By Proposition 5.1, we only need to prove (5.6).

Let $N$ be the $C^{2}$ vector field on $\bar{\Omega}$ satisfying (3.4). Multiply both sides of the first equation of (5.5) by $N\left(\bar{v}_{\varepsilon}\right)$ and integrate over $\Omega$. Using Green's first formula and the fact that $N\left(\bar{v}_{\varepsilon}\right)=\left\langle\nabla_{g} \bar{v}_{\varepsilon}, N\right\rangle_{g}=\frac{\partial \bar{v}_{\varepsilon}}{\partial \mu}$ on $\Gamma$, we obtain

$$
\begin{aligned}
0 & =\int_{\Omega} \varepsilon^{-1} v_{\varepsilon} N\left(\bar{v}_{\varepsilon}\right) d x-i \int_{\Omega} P v_{\varepsilon} N\left(\bar{v}_{\varepsilon}\right) d x \\
& =\varepsilon^{-1} \int_{\Omega} v_{\varepsilon} N\left(\bar{v}_{\varepsilon}\right) d x-i \int_{\Omega}\left(-\Delta_{g}+D q\right) v_{\varepsilon} N\left(\bar{v}_{\varepsilon}\right) d x \\
& =\varepsilon^{-1} \int_{\Omega} v_{\varepsilon} N\left(\bar{v}_{\varepsilon}\right) d x+i \int_{\Gamma}\left|\frac{\partial v_{\varepsilon}}{\partial \mu}\right|^{2} d \Gamma-i \int_{\Omega}\left\langle\nabla_{g} v_{\varepsilon}, \nabla_{g} N\left(\bar{v}_{\varepsilon}\right)\right\rangle_{g} d x \\
& -i \int_{\Omega} D q\left(v_{\varepsilon}\right) N\left(\bar{v}_{\varepsilon}\right) d x
\end{aligned}
$$

It then follows that

$$
\begin{aligned}
\int_{\Gamma}\left|\frac{\partial v_{\varepsilon}}{\partial \mu}\right|^{2} d \Gamma & =\operatorname{Re} \int_{\Omega}\left\langle\nabla_{g} v_{\varepsilon}, \nabla_{g} N\left(\bar{v}_{\varepsilon}\right)\right\rangle_{g} d x-\varepsilon^{-1} \operatorname{Im} \int_{\Omega} v_{\varepsilon} N\left(\bar{v}_{\varepsilon}\right) d x \\
& +\operatorname{Re} \int_{\Omega} D q\left(v_{\varepsilon}\right) N\left(\bar{v}_{\varepsilon}\right) d x
\end{aligned}
$$

Substitute (3.6) into (5.11), and make use of the divergence theorem, (3.8) and Lemma 2.3 to give

$$
\begin{aligned}
\int_{\Gamma}\left|\frac{\partial v_{\varepsilon}}{\partial \mu}\right|^{2} d \Gamma= & 2 \operatorname{Re} \int_{\Omega} D N\left(\nabla_{g} v_{\varepsilon}, \nabla_{g} \bar{v}_{\varepsilon}\right) d x-\int_{\Omega}\left|\nabla_{g} v_{\varepsilon}\right|_{g}^{2} \operatorname{div}_{g}(N) d x \\
& -2 \varepsilon^{-1} \operatorname{Im} \int_{\Omega} v_{\varepsilon} N\left(\bar{v}_{\varepsilon}\right) d x+2 \operatorname{Re} \int_{\Omega} D q\left(v_{\varepsilon}\right) N\left(\bar{v}_{\varepsilon}\right) d x \\
\leq & C \int_{\Omega}\left|\nabla_{g} v_{\varepsilon}\right|_{g}^{2} d x-2 \varepsilon^{-1} \operatorname{Im} \int_{\Omega} v_{\varepsilon} N\left(\bar{v}_{\varepsilon}\right) d x \\
\leq & C \int_{\Omega}\left|\nabla_{g} v_{\varepsilon}\right|_{g}^{2} d x+\varepsilon^{-3 / 2} \int_{\Omega}\left|v_{\varepsilon}\right|^{2} d x+\varepsilon^{-1 / 2} \int_{\Omega}\left|\nabla_{g} v_{\varepsilon}\right|_{g}^{2} d x
\end{aligned}
$$

where $C$ is a positive constant independent of $\varepsilon$.

Next, multiply both sides of the first equation of (5.5) by $\bar{v}_{\varepsilon}$ and integrate over $\Omega$ by parts to obtain

$$
\begin{aligned}
0 & =\varepsilon^{-1} \int_{\Omega}\left|v_{\varepsilon}\right|^{2} d x-i \int_{\Omega} P v_{\varepsilon} \bar{v}_{\varepsilon} d x \\
& =\varepsilon^{-1} \int_{\Omega}\left|v_{\varepsilon}\right|^{2} d x+i \int_{\Gamma} \frac{\partial v_{\varepsilon}}{\partial \nu_{\mathcal{A}}} \bar{u} d \Gamma-i \int_{\Omega}\left|\nabla_{g} v_{\varepsilon}\right|_{g}^{2} d x .
\end{aligned}
$$

Compare the imaginary part of the identity (5.13) and multiply by $\varepsilon^{3 / 2}$ to give

$$
\varepsilon^{3 / 2} \int_{\Omega}\left|\nabla_{g} v_{\varepsilon}\right|_{g}^{2} d x \leq \frac{\varepsilon^{1 / 2}}{2} \int_{\Gamma}|u|^{2} d \Gamma+\frac{\varepsilon^{5 / 2}}{2} \int_{\Gamma}\left|\frac{\partial v_{\varepsilon}}{\partial \nu_{\mathcal{A}}}\right|^{2} d \Gamma
$$


The same treatment to the real part of the identity (5.13) yields

$$
\varepsilon^{1 / 2} \int_{\Omega}\left|v_{\varepsilon}\right|^{2} d x \leq \frac{\varepsilon^{1 / 2}}{2} \int_{\Gamma}|u|^{2} d \Gamma+\frac{\varepsilon^{5 / 2}}{2} \int_{\Gamma}\left|\frac{\partial v_{\varepsilon}}{\partial \nu_{\mathcal{A}}}\right|^{2} d \Gamma
$$

Combining (5.12), (5.14), and (5.15), and making use of (3.8) and (3.9), we obtain

$$
\begin{aligned}
\int_{\Gamma}\left|\varepsilon \frac{\partial v_{\varepsilon}}{\partial \nu_{\mathcal{A}}}\right|^{2} d \Gamma & \leq C b \varepsilon^{2} \int_{\Omega}\left|\nabla_{g} v_{\varepsilon}\right|_{g}^{2} d x+b \varepsilon^{1 / 2} \int_{\Gamma}|u|^{2} d \Gamma \\
& +b \varepsilon^{1 / 2} \int_{\Gamma}\left|\varepsilon \frac{\partial v_{\varepsilon}}{\partial \nu_{\mathcal{A}}}\right|^{2} d \Gamma \leq\left(C b \frac{\varepsilon}{2}+b \varepsilon^{1 / 2}\right) \int_{\Gamma}|u|^{2} d \Gamma \\
& +\left(\frac{C b}{2} \varepsilon^{1 / 2}+b\right) \varepsilon^{1 / 2} \int_{\Gamma}\left|\varepsilon \frac{\partial v_{\varepsilon}}{\partial \nu_{\mathcal{A}}}\right|^{2} d \Gamma
\end{aligned}
$$

This implies that

The proof is complete.

$$
\lim _{\varepsilon \rightarrow 0+} \int_{\Gamma}\left|\varepsilon \frac{\partial v_{\varepsilon}}{\partial \nu_{\mathcal{A}}}\right|^{2} d \Gamma=0
$$

Proposition 5.2. Theorem 1.5 is valid if for any $u \in C_{0}^{\infty}(\Gamma)$ and $\varepsilon>0$, the solution $w_{\varepsilon}$ to the equation

satisfies

$$
\begin{cases}w_{\varepsilon}(x)+\varepsilon^{2} P^{2} w_{\varepsilon}(x)=0, & x \in \Omega, \\ w_{\varepsilon}(x)=0, P w_{\varepsilon}(x)=u(x), & x \in \Gamma\end{cases}
$$

$$
\lim _{\varepsilon \rightarrow 0+} \int_{\Gamma}\left|\varepsilon \frac{\partial\left(P w_{\varepsilon}\right)}{\partial \nu_{\mathcal{A}}}\right|^{2} d \Gamma=0
$$

Proof. As in the beginning of proof of Proposition 5.1, (1.7) is equivalent to, in the strong topology of $L^{2}(\Gamma)$, that

$$
\lim _{\lambda \in \mathbb{R}, \lambda \rightarrow+\infty} H(\lambda) u=0, \forall u \in L^{2}(\Gamma),
$$

where $H(\lambda)$ is given by (5.2). Due to (5.4) and a density argument, it suffices to show that (5.19) is satisfied for all $u \in C_{0}^{\infty}(\Gamma)$. Now let $u \in C_{0}^{\infty}(\Gamma)$. Denote by

$$
w_{\lambda}(x)=\left(\left(\lambda^{2}+\tilde{A}\right)^{-1} B u\right)(x), \forall x \in \Omega .
$$

Then $w_{\lambda}(x)$ satisfies

$$
\begin{cases}\lambda^{2} w_{\lambda}(x)+P^{2} w_{\lambda}(x)=0, & x \in \Omega, \\ w_{\lambda}(x)=0, P w_{\lambda}(x)=u(x), & x \in \Gamma,\end{cases}
$$

and the transfer function $H(\lambda)$ can be written as

$$
(H(\lambda) u)(x)=\lambda B^{*} w_{\lambda}=\lambda \frac{\partial \mathcal{A}^{-1} w_{\lambda}}{\partial \nu_{\mathcal{A}}}(x), \forall x \in \Gamma .
$$

Since $u \in C_{0}^{\infty}(\Gamma)$, there exists a unique classical solution to (5.20). Let $\tilde{w} \in C^{\infty}(\Omega)$ be the unique solution to the following boundary value problem

$$
\begin{cases}P^{2} \tilde{w}(x)=0, & x \in \Omega, \\ \tilde{w}(x)=0, P \tilde{w}(x)=u(x), & x \in \Gamma .\end{cases}
$$


Then (5.20) becomes

$$
\begin{cases}\lambda^{2} w_{\lambda}(x)+P^{2}\left(w_{\lambda}(x)-\tilde{w}(x)\right)=0, & x \in \Omega, \\ w_{\lambda}(x)-\tilde{w}(x)=0, P\left(w_{\lambda}(x)-\tilde{w}(x)\right)=0, & x \in \Gamma,\end{cases}
$$

or equivalently,

$$
\lambda^{2} \mathcal{A}^{-1} w_{\lambda}=P w_{\lambda}-P \tilde{w}
$$

Substitute (5.22) into (5.21), to obtain

$$
(H(\lambda) u)(x)=\frac{1}{\lambda} \frac{\partial\left(P w_{\lambda}\right)}{\partial \nu_{\mathcal{A}}}(x)-\frac{1}{\lambda} \frac{\partial(P \tilde{w})}{\partial \nu_{\mathcal{A}}}(x) .
$$

Since $P \tilde{w}$ is independent of $\lambda$, letting $w_{\varepsilon}(x)=w_{\lambda}(x)$ with $\varepsilon=1 / \lambda$, we conclude the required result.

Proof of Theorem 1.5. By Proposition 5.2, it suffices to prove (5.18).

We decompose $u$ into the real part and the imaginary part. Let $u=u_{1}+i u_{2}$, where $u_{1}$ and $u_{2}$ are real functions in $C_{0}^{\infty}(\Gamma)$. Then the solution $w_{\varepsilon}$ to (5.17) can be uniquely decomposed as $w_{\varepsilon}=w_{1 \varepsilon}+i w_{2 \varepsilon}$ corresponding to the decomposition of $u$, where the real function $w_{k \varepsilon}$ verifies the equation

$$
\begin{cases}w_{k \varepsilon}(x)+\varepsilon^{2} P^{2} w_{k \varepsilon}(x)=0, & x \in \Omega, \\ w_{k \varepsilon}(x)=0, P w_{k \varepsilon}(x)=u_{k}(x), & x \in \Gamma,\end{cases}
$$

for $k=1,2$. Then (5.18) is equivalent to

$$
\lim _{\varepsilon \rightarrow 0+} \int_{\Gamma}\left|\varepsilon \frac{\partial\left(P w_{k \varepsilon}\right)}{\partial \nu_{\mathcal{A}}}\right|^{2} d \Gamma=0, \forall k=1,2 .
$$

Therefore, it is sufficient to show (5.18) for real functions $u \in C_{0}^{\infty}(\Gamma)$. Now assume that $u \in C_{0}^{\infty}(\Gamma)$ is a real function. Denote by

$$
\varphi_{\varepsilon}(x)=\varepsilon^{-1} w_{\varepsilon}(x)+i P w_{\varepsilon}(x), \forall x \in \Omega .
$$

Then $\varphi_{\varepsilon}$ satisfies the equation

$$
\begin{cases}\varepsilon^{-1} \varphi_{\varepsilon}(x)-i P \varphi_{\varepsilon}(x)=0, & x \in \Omega, \\ \varphi_{\varepsilon}(x)=-i u(x), & x \in \Gamma .\end{cases}
$$

While (5.25) is the same type with that of (5.5). It follows from (5.6) and (5.24) that

$$
0=\lim _{\varepsilon \rightarrow 0+} \int_{\Gamma}\left|\varepsilon \frac{\partial \varphi_{\varepsilon}}{\partial \nu_{\mathcal{A}}}\right|^{2} d \Gamma=\lim _{\varepsilon \rightarrow 0+} \int_{\Gamma}\left|\frac{\partial w_{\varepsilon}}{\partial \nu_{\mathcal{A}}}\right|^{2} d \Gamma+\lim _{\varepsilon \rightarrow 0+} \int_{\Gamma}\left|\varepsilon \frac{\partial\left(P w_{\varepsilon}\right)}{\partial \nu_{\mathcal{A}}}\right|^{2} d \Gamma .
$$

Therefore, (5.18) is valid. The proof is complete.

\section{REFERENCES}

[1] K. Ammari, Dirichlet boundary stabilization of the wave equation, Asymptot. Anal., 30 (2002), 117-130. MR.1919338 (2003f:93072)

[2] K. Ammari, M. Tucsnak, Stabilization of second-order evolution equations by a class of unbounded feedbacks, ESAIM Control Optim. Calc. Var., 6 (2001), 361-386. MR 1836048 (2002f:93104)

[3] S. G. Chai and B. Z. Guo, Feedthrough operator for linear elasticity system with boundary control and observation, SIAM Journal on Control and Optimization, 48(2010), 3708-3734. MR 2606833

[4] S. G. Chai and B. Z. Guo, Well-posedness and regularity of Naghdi's shell equation under boundary control and observation, J. Differential Equations, 249(2010), 3174-3214. MR2737426 
[5] C. I. Byrnes, D. S. Gilliam, V. I. Shubov and G. Weiss, Regular linear systems governed by a boundary controlled heat equation, J. Dyn. Control Syst., 8 (2002), 341-370. MR.1914447 (2003d:93045)

[6] R. F. Curtain, The Salamon-Weiss class of well-posed infinite dimensional linear systems: A survey, IMA J. Math. Control Inform., 14 (1997), 207-223. MR.1470034 (99a:93054)

[7] R. F. Curtain, Linear operator inequalities for strongly stable weakly regular linear systems, Math. Control Signals Syst., 14 (2001), 299-337. MR1868533 (2002k:93022)

[8] R. F. Curtain and G. Weiss, Well-posedness of triples of operators (in the sense of linear systems theory) in "Control and Estimation of Distributed Parameter Systems" (F. Kappel, K. Kunisch and W. Schappacher, Eds.), Vol. 91, Birkhäuser, Basel, 1989, 41-59. MR1033051(91d:93027)

[9] P. Grisvard, A Caractérization de quelques espaces d'interpolation, Arch. Rational Mech. Anal., 25 (1967), 40-63. MR0213864 (35:4718)

[10] B. Z. Guo and Y. H. Luo, Controllability and stability of a second order hyperbolic system with collocated sensor/actuator, Systems Control Lett., 46 (2002), 45-65. MR2011071 (2004i:93015)

[11] B. Z. Guo and Z. C. Shao, Regularity of a Schrödinger equation with Dirichlet control and colocated observation, Systems Control Lett., 54 (2005), 1135-1142. MR2170295 (2006d:35208)

[12] B. Z. Guo and Z. C. Shao, Regularity of an Euler-Bernoulli plate equation with Neumann control and collocated observation, J. Dyn. Control Syst., 12 (2006), 405-418. MR.2233027 (2007b:93076)

[13] B. Z. Guo and Z. C. Shao, On well-posedness, regularity and exact controllability for problems of transmission of plate equation with variable coefficients, Quart. Appl. Math., 65 (2007), 705-736. MR2370357(2009a:93014)

[14] B. Z. Guo and X. Zhang, The regularity of the wave equation with partial Dirichlet control and collocated observation, SIAM J. Control Optim., 44 (2005), 1598-1613. MR.2193497 (2006j:93058)

[15] B. Z. Guo and Z. X. Zhang, On the well-posedness and regularity of wave equations with variable coefficients and partial boundary Dirichlet control and colocated observation, ESAIM Control Optim. Calc. Var., 13 (2007), 776-792. MR2351403(2009a:35153)

[16] B. Z. Guo and Z. X. Zhang, Well-Posedness and regularity for an Euler-Bernoulli plate with variable coefficients and boundary control and observation, Math. Control Signals Syst., 19 (2007), 337-360. MR2354054 (2009g:93008)

[17] B. Z. Guo and Z. X. Zhang, Well-posedness of systems of linear elasticity with Dirichlet boundary control and observation, SIAM Journal on Control and Optimization, 48 (2009), 2139-2167. MR 2520323 (2010h:93049)

[18] V. Komornik, Exact Controllability and Stabilization: The Multiplier Method, Wiley, Chichester, 1994. MR.1359765 (96m:93003)

[19] I. Lasiecka, Exponential decay rates for the solutions of Euler-Bernoulli equations with boundary dissipation occurring in the moments only, J. Differential Equations, 95 (1992), 169-182. MR.1142282 (92k:35073)

[20] J. E. Lagnese and J. L. Lions, Modelling, Analysis and Control of Thin Plate, Collection RMA, Masson, Paris, 1988. MR953313 (89k:73001)

[21] J. L. Lions and E. Magenes, Non-Homogeneous Boundary Value Problems and Applications, Vol. I, II, Springer-Verlag, Berlin, 1972. MR0350177 (50:2670)

[22] I. Lasiecka and R. Triggiani, $L^{2}(\Sigma)$-regularity of the boundary to boundary operator $B^{*} L$ for hyperbolic and Petrowski PDEs, Abstr. Appl. Anal., 2003:19 (2003), 1061-1139. MR2041290 (2005i:35164)

[23] I. Lasiecka and R. Triggiani, The operator $B^{*} L$ for the wave equation with Dirichlet control, Abstr. Appl. Anal., 2004:7 (2004), 625-634. MR2084941(2005i:35162)

[24] S. E. Rebiai, Uniform energy decay of Schrödinger equations with variable coefficients, IMA $J$. Math. Control Inform., 20 (2003), 335-345. MR2009070(2004i:35066)

[25] D. Salamon, Infinite dimensional systems with unbounded control and observation: a functional analytic approach, Trans. Amer. Math. Soc., 300 (1987), 383-481. MR876460 (88d:93024)

[26] D. Salamon, Realization theory in Hilbert space, Math. Systems Theory, 21 (1989), 147-164. MR.977021 (89k:93038)

[27] O. J. Staffans, Passive and conservative continuous-time impedance and scattering systems. Part I: well-posed systems, Math. Control Signals Syst., 15 (2002), 291-315. MR1942089 (2003i:93024)

[28] M. E. Taylor, Partial Differential Equations I: Basic Theory, Springer-Verlag, New York, 1996. MR 1395148 (98b:35002b)

[29] G. Weiss, Admissible observation operators for linear semigroups, Israel J. Math., 65 (1989), no. 1, 17-43. MR994732 (90g:47082) 
[30] G. Weiss, Admissibility of unbounded control operators, SIAM J. Control Optim., 27 (1989), 527545. MR993285 (90c:93060)

[31] G. Weiss, The representation of regular linear systems on Hilbert spaces, Control and estimation of distributed parameter systems (Vorau, 1988), 401-416, Internat. Ser. Numer. Math., 91, Birkhäuser, Basel, 1989. MR1033074 (91d:93026)

[32] G. Weiss, Transfer functions of regular linear systems I: characterizations of regularity, Trans. Amer. Math. Soc., 342 (1994), 827-854. MR1179402 (94f:93074)

[33] P. F. Yao, On the observatility inequality for exact controllability of wave equations with variable coefficients, SIAM J. Control Optim., 37 (1999), 1568-1599. MR.1710233 (2000m:93027)

[34] P. F. Yao, Observability inequalities for the Euler-Bernoulli plate with variable coefficients, Contemporary Mathematics, Amer. Math. Soc., Providence, RI, 268 (2000), 383-406. MR1804802 (2001m:93025) 